\title{
Resolving the inner regions of the HD 97048 circumstellar disk with VLT/NACO polarimetric differential imaging ${ }^{\star}$
}

\author{
S. P. Quanz ${ }^{1}$, S. M. Birkmann ${ }^{2}$, D. Apai ${ }^{3}$, S. Wolf ${ }^{4}$, and T. Henning ${ }^{5}$ \\ ${ }^{1}$ Institute for Astronomy, ETH Zurich, Wolfgang-Pauli-Strasse 27, 8093 Zurich, Switzerland \\ e-mail: quanz@astro.phys.ethz.ch \\ 2 ESA/ESTEC, Keplerlaan 1, Postbus 299, 2200 AG Noordwijk, The Netherlands \\ 3 Department of Astronomy/Department of Planetary Sciences, University of Arizona, 933 N. Cherry Ave., Tucson, AZ 85721, USA \\ ${ }^{4}$ University of Kiel, Institute of Theoretical Physics and Astrophysics, Leibnizstrasse 15, 24098 Kiel, Germany \\ 5 Max Planck Institute for Astronomy, Königstuhl 17, 69117 Heidelberg, Germany
}

Received 11 October 2011 / Accepted 7 December 2011

\begin{abstract}
Context. Circumstellar disks are the cradles of planetary systems and their physical and chemical properties directly influence the planet formation process. Because most planets supposedly form in the inner disk regions, i.e., within a few tens of AU, it is crucial to study circumstellar disks on these scales to constrain the conditions for planet formation.

Aims. Our aims are to characterize the inner regions of the circumstellar disk around the young Herbig Ae/Be star HD 97048 in polarized light.

Methods. We used VLT/NACO to observe HD 97048 in polarimetric differential imaging (PDI) mode in the $H$ and $K_{\mathrm{s}}$ band. This technique offers high-contrast capabilities at very small inner working angles and probes the dust grains on the surface layer of the disk that act as the scattering surface.

Results. We spatially resolve the disk around HD 97048 in polarized flux in both filters on scales between $\sim 0.1^{\prime \prime}-1.0^{\prime \prime}$ corresponding to the inner $\sim 16-160$ AU. Fitting isophots to the flux calibrated $H$-band image between 13-14 mag/arcsec ${ }^{2}$ and $14-15 \mathrm{mag} / \mathrm{arcsec}^{2}$, we derive an apparent disk inclination angle of $34^{\circ} \pm 5^{\circ}$ and $47^{\circ} \pm 2^{\circ}$, respectively. The disk position angle in both brightness regimes is almost identical and roughly $80^{\circ}$. Along the disk major axis the surface brightness of the polarized flux drops from $\sim 11 \mathrm{mag} / \mathrm{arcsec}^{2}$ at $\sim 0.1^{\prime \prime}(\sim 16 \mathrm{AU})$ to $\sim 15.3 \mathrm{mag} / \operatorname{arcsec}^{2}$ at $\sim 1.0^{\prime \prime}(\sim 160 \mathrm{AU})$. The brightness profiles along the major axis are fitted with power-laws falling off as $\propto r^{-1.78 \pm 0.02}$ in $H$ and $\propto r^{-2.34 \pm 0.04}$ in $K_{\mathrm{s}}$. Because the surface brightness decreases more rapidly in $K_{\mathrm{s}}$ compared to $H$, the disks becomes relatively bluer at larger separations, possibly indicating changing dust grain properties as a function of radius.

Conclusions. We imaged for the first time the inner $\sim 0.1^{\prime \prime}-1.0^{\prime \prime}(\sim 16-160 \mathrm{AU})$ of the surface layer of the HD 97048 circumstellar disk in scattered light, which demonstrates the power of ground-based imaging polarimetry. Our data fill an important gap in a large collection of existing data that include resolved thermal dust and polycyclic aromatic hydrocarbon (PAH) emission and also resolved gas emission lines. HD 97048 therefore is an ideal test case for sophisticated models of circumstellar disks and a prime target for future high-contrast imaging observations.
\end{abstract}

Key words. methods: observational - stars: formation - stars: pre-main sequence - protoplanetary disks - techniques: polarimetric

\section{Introduction}

Planets form in disks of gas and dust around young stars, and models show that the physical and chemical properties of these disks influence the outcome of the planet formation process (e.g., Alibert et al. 2011). At least for sun-like stars most direct imaging surveys for exoplanets found that (massive) planets on large orbital separations ( $\gtrsim 50 \mathrm{AU})$ are fairely scarce (e.g., Masciadri et al. 2005; Biller et al. 2007; Kasper et al. 2007; Apai et al. 2008; Lafrenière et al. 2007; Chauvin et al. 2010; Janson et al. 2011) but radial velocity and transit searches have demonstrated that the inner few $\mathrm{AU}$ are packed with (low-mass) planets (e.g., Mayor et al. 2011; Borucki et al. 2011). The direct detection of giant planets orbiting between $\sim 10-70$ AU around the A-type stars $\beta$ Pictoris (Lagrange et al. 2009; Lagrange et al. 2010; Quanz et al. 2010) and HR8799 (Marois et al. 2008, 2010) suggest that at least intermediate-mass stars can form planets out

\footnotetext{
* Based on observations collected at the European Organisation for Astronomical Research in the Southern Hemisphere, Chile (program number: 077.C-0106A).
}

to a few tens of $\mathrm{AU}$. Thus, one requires an observing technique capable of resolving those inner regions of circumstellar disks where planets are potentially forming.

Polarimetric differential imaging (PDI) is a technique that allows high-contrast direct imaging of circumstellar disks at very small inner working angles (Kuhn et al. 2001; Potter 2003; Apai et al. 2004; Perrin et al. 2004). It takes advantage of the fact that direct stellar light is largely unpolarized while stellar photons that scatter of the dust grains on the surface layer of a circumstellar disk are polarized. In a recent paper we presented nearinfrared (NIR) PDI observations of the circumstellar disk around the Herbig Ae/Be star 100546 (Quanz et al. 2011). These observations revealed disk structures, geometry, and dust grain properties as close as $0.1^{\prime \prime}$ to the central star, i.e., regions that are difficult to probe by other direct imaging techniques in the NIR such as coronagraphy or classical point spread function (PSF) subtraction.

In this paper we present PDI data of HD 97048, another well-studied Herbig Ae/Be star. The key stellar parameters are summarized in Table 1. We note that throughout this paper we 
Table 1. Properties of central star.

\begin{tabular}{lcc}
\hline \hline Parameter & HD 97048 & Reference \\
\hline RA $(\mathrm{J} 2000)$ & $11^{\mathrm{h}} 08^{\mathrm{m}} 03.32^{\mathrm{s}}$ & $(1)$ \\
Dec $(\mathrm{J} 2000)$ & $-77^{\circ} 39^{\prime} 17.48^{\prime \prime}$ & $(1)$ \\
$H[\mathrm{mag}]$ & 6.67 & $(2)$ \\
$K_{\mathrm{s}}[\mathrm{mag}]$ & 5.94 & $(2)$ \\
Distance [pc] & $158_{-14}^{+16}$ & $(1)$ \\
Sp. Type & $\mathrm{B} 9-\mathrm{A} 0 \mathrm{ep}+\mathrm{sh}$ & $(3)$ \\
Age $[\mathrm{Myr}]$ & $>2,3$ & $(4),(5)$ \\
log $\left(T_{\mathrm{eff}}\right)[\mathrm{K}]$ & 4.0 & $(4)$ \\
$A_{V}[\mathrm{mag}]$ & 1.24 & $(4)$ \\
Mass $\left[M_{\odot}\right]$ & $2.5 \pm 0.2$ & $(4)$ \\
\hline
\end{tabular}

References. (1) van Leeuwen (2007); (2) Cutri et al. (2003); (3) Whittet et al. (1987); (4) van den Ancker et al. (1998); (5) Lagage et al. (2006).

assumed a distance of $158_{-14}^{+16} \mathrm{pc}$ for HD 97048 as derived from a re-analysis of the Hipparcos measurements by van Leeuwen (2007). Most earlier studies used the initial Hipparcos results of $180_{-20}^{+30} \mathrm{pc}$ found by van den Ancker et al. (1997) ${ }^{1}$.

HD 97048 is surrounded by a circumstellar disk that was clearly resolved in mid-infrared (MIR) images taken in polycyclic aromatic hydrocarbon (PAH) filters (Lagage et al. 2006; Doucet et al. 2007). Spectroscopic studies in the MIR also showed very strong and resolved PAH emission bands but no silicate emission feature at $10 \mu \mathrm{m}$ (Van Kerckhoven et al. 2002; van Boekel et al. 2004). Doering et al. (2007) imaged the circumstellar surrounding of HD 97048 with HST/ACS in the F606W (broad $V$ ) filter and detected scattered light out to a radial distance of $\sim 4^{\prime \prime}$ in almost all directions. The inner $2^{\prime \prime}$ (in radius) were excluded from any analysis because of the occulting spot used for the observations and substantial subtraction residuals. Single-dish observations of the mm continuum emission suggest a circumstellar mass of $\sim 0.2 M_{\odot}$ (Henning et al. 1998) but part of this mass is probably residing in an envelope, also seen in extended MIR emission (Prusti et al. 1994; Siebenmorgen et al. 2000), surrounding the star-disk system. Several gas emission lines ([OI], $\mathrm{CO}$ and $\mathrm{H}_{2}$ ) have been detected and were, at least partly, spectrally and spatially resolved (Acke \& van den Ancker 2006; van der Plas et al. 2009; Martin-Zaïdi et al. 2007, 2009; Carmona et al. 2011).

Our polarimetric images resolved the inner parts of the HD 97048 circumstellar disk for the first time in scattered light in the $H$ and $K_{\mathrm{s}}$ band. The observations and data reduction are described in Sect. 2, the key results and main analyses are presented in Sect. 3, and we discuss our results in broader context in Sect. 4. Finally, we conclude in Sect. 5.

\section{Observations and data reduction}

The observations were carried out with VLT/NACO (Lenzen et al. 2003; Rousset et al. 2003) on ESO's UT4 in April 2006 and followed the same strategy as those used in Quanz et al. (2011). The SL27 camera with a pixel scale of $\sim 27$ mas/pixel was used with the detector set to HighDynamic mode and read

\footnotetext{
1 Already Whittet et al. (1997) favored a distance of only $160 \mathrm{pc}$ consistent with the distance to the Chameleon I region. We emphasize, however, that the error bars for both derived distances overlap and that the only figure in this paper that directly depends on the exact value for the distance is the projected separation of circumstellar material around the star.
}

out in Double RdRstRd mode. For our polarimetric observations the Wollaston prism was used, which splits the light into the ordinary and extraordinary beam offset by $3.5^{\prime \prime}$ in $y$-direction on the detector. The polarimetric mask avoids overlap of the two images on the detector but restricts the original field of view of $27^{\prime \prime} \times 27^{\prime \prime}$ to $x=27^{\prime \prime}$ long and $y=3.1^{\prime \prime}$ wide stripes separated by $3.5^{\prime \prime}$ in $y$-direction. To obtain full polarization cycles we used the rotatable half-wave retarder plate at four different positions $\left(0.0^{\circ},-22.5^{\circ},-45.0^{\circ},-67.5^{\circ}\right)$. The object was moved along the detector's $x$-axis between consecutive polarization cycles to correct for bad pixels.

We observed HD 97048 in three different filters $\left(H, K_{\mathrm{s}}\right.$, $N B 1.64)$. The core of the PSF was saturated in the $H$ and $K_{\mathrm{s}}$ filter so that the inner 5-6 pixels (in diameter) were no longer in the linear detector regime (i.e. $>10000$ counts). The vast majority of the frames taken in the narrow band filter were unsaturated and only individual frames showed count rates slightly above the linearity threshold. The FWHM of the PSF in the NB1.64 was typically $4.0-4.5$ pixels (i.e., $0.11^{\prime \prime}-0.12^{\prime \prime}$ ) wide. Table 2 summarizes the observations and the observing conditions.

The data reduction was laid out in Quanz et al. (2011) and we refer the reader to this paper for a very detailed description. In short, after basic image processing all images were aligned with sub-pixel accuracy and for each dither position we computed the fractional polarization images $p_{Q}$ and $p_{U}$ in both filters. Averaging over all dither positions gave us the final images $\overline{p_{Q}}$ and $\overline{p_{U}}$. To obtain the final Stokes $Q$ and $U$ images we computed the average intensity images $I$ for each filter and multiplied $Q=\overline{p_{Q}} I$ and $U=\overline{p_{U}} I$. The total polarized flux images $P$ were then derived via $P=\left(Q^{2}+U^{2}\right)^{\frac{1}{2}}$.

Also identical to the approach explained in Quanz et al. (2011), the instrumental polarization was determined and corrected for before the final fractional polarization images were computed. We found the following offsets: 0.01102 and -0.00770 in the $H$ band for $p_{Q}$ and $p_{U}$, respectively, and 0.00018 and -0.00193 for the corresponding parameters in the $K_{\mathrm{s}}$ filter. Finally, we were only able to flux calibrate the final $P$ image in the $H$ band because we only obtained unsaturated images in the NB1.64 filter (see, Quanz et al. 2011). We estimate that the photometric calibration of the final $H$ band image is good to $40-50 \%$ given the uncertainties in the instrumental polarization and in the calibration itself.

\section{Results and analysis}

In Fig. 1 we show the final Stokes $Q$ and $U$ images of HD 97048 in the $H$ filter. The expected "butterfly" pattern of a circumstellar disk observed in PDI is apparent with the pattern being rotated by $\sim 45^{\circ}$ between $Q$ and $U$. Stokes $Q$ is normally defined to be positive in the north-south direction, but as explained in Witzel et al. (2011) and Quanz et al. (2011), there was an offset of $\sim 13.2^{\circ}$ in the encoder position of NACO's half-wave plate compared to the actual position on the sky. Therefore, the pattern in Fig. 1 is rotated by this amount counterclockwise.

In Fig. 2 we show the final total polarized flux images in the $H$ and $K_{\mathrm{s}}$ band. The $H$-band image is flux calibrated and the surface brightness of the disk is given in mag/arcsec ${ }^{2}$. Because the general morphology and radial extent of the two images is quite similar, we are confident that we have indeed imaged the surface layer of a dusty circumstellar disk. To first order we did not find significant asymmetries in the flux distribution in both filters. Only in the northwest quadrant (roughly at $x=0.4^{\prime \prime}$ and $\left.y=0.4^{\prime \prime}\right)$ of the $H$-band image appears to be a flux excess 
Table 2. Summary of observations and observing conditions.

\begin{tabular}{lccccccc}
\hline \hline Object & Filter & DIT $\times$ NDIT $^{a}$ & $\begin{array}{c}\text { Dither } \\
\text { positions }^{b}\end{array}$ & Airmass & Obs. date & $\begin{array}{c}\langle E C\rangle^{c}[\%] \\
\text { mean/min/max }\end{array}$ & $\begin{array}{c}\left\langle\tau_{0}\right\rangle^{d}[\mathrm{~ms}] \\
\operatorname{mean} / \mathrm{min} / \mathrm{max}\end{array}$ \\
\hline HD 97048 & $H$ & $0.35 \mathrm{~s} \times 85$ & $10(12)$ & $1.76-1.68$ & $2006-A p r .-08$ & $42.3 / 14.9 / 56.7$ & $5.9 / 3.7 / 9.4$ \\
& $K_{\mathrm{s}}$ & $0.35 \mathrm{~s} \times 85$ & $8(8)$ & $1.68-1.66$ & 2006-Apr.-08 & $50.2 / 41.3 / 55.9$ & $5.5 / 2.7 / 8.1$ \\
& $N B 1.64$ & $3.0 \mathrm{~s} \times 20$ & $16(16)$ & $1.66-1.74$ & 2006-Apr.-08 & $53.9 / 46.2 / 60.4$ & $7.3 / 3.0 / 9.8$ \\
\hline
\end{tabular}

Notes. ${ }^{a}$ Detector integration time (DIT) $\times$ number of integrations (NDIT), i.e., total integration time per dither position and per retarder plate position; ${ }^{b}$ Number of dither positions used in final analysis and in parenthesis total number of observed dither position. The difference was disregarded because of poor AO correction. At each dither position NDIT exposures were taken at each of the four different retarder plate positions $\left(0.0^{\circ},-22.5^{\circ},-45.0^{\circ},-67.5^{\circ}\right) ;^{c}$ Average, minimum and maximum value of the coherent energy of the PSF. Calculated by the Real Time Computer of the AO system; ${ }^{d}$ Average, minimum and maximum value of the coherence time of the atmosphere. Calculated by the Real Time Computer of the AO system.
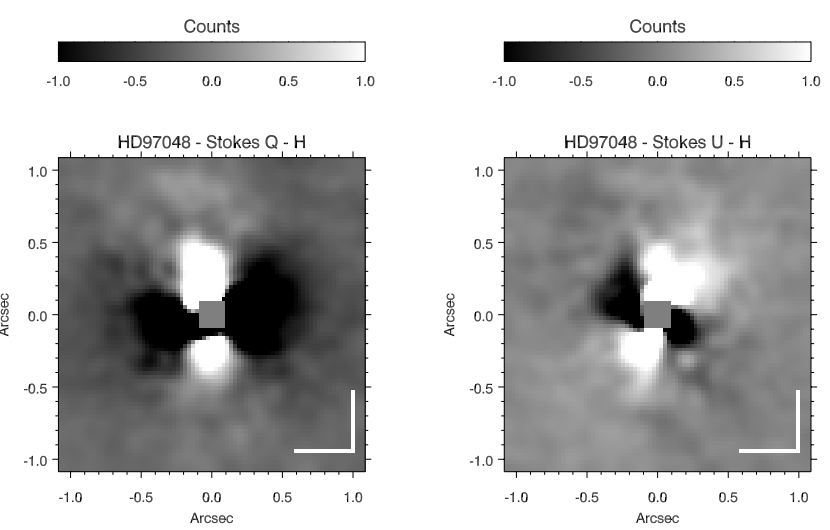

Fig. 1. Final Stokes $Q$ (left) and Stokes $U$ (right) images of HD 97048 in the $H$ filter. The innermost saturated pixels have been masked out and the images have been convolved with a Gaussian kernel with a FWHM of 4 pixels (i.e., the FWHM of the PSF). The expected "butterfly" pattern is clearly detected. The units are given in counts per pixel. North is up, east to the left.

compared to the corresponding position in the northeast quadrant. In the $K_{\mathrm{s}}$-filter image there seems to be a filamentary-like, elongated structure in the northern part of the disk stretching from $\sim 0.5-1.0^{\prime \prime}$. However, given that we do not see the same feature in the $H$-filter image and that the total integration time in the $K_{\mathrm{s}}$ image was shorter and hence the signal-to-noise lower, it is unclear whether this feature is real.

To estimate the disk inclination we fitted ellipses to isophots in the images shown in Fig. 2. While this approach is widely used in the literature, it does not take into account potential disk flaring and the scattering (and polarizing) properties of the dust grains. Therefore, one has to keep in mind that fitting disk regions of the same brightness does not necessarily mean that one fits the same physical regions. However, this approach gives a first indication about the apparent disk orientation. In the $H$-band image we fitted one ellipse to regions where the surface brightness is between $13-14 \mathrm{mag} / \operatorname{arcsec}^{2}$ and one where it is between $14-15 \mathrm{mag} / \operatorname{arcsec}^{2}$. For the brighter regions the apparent inclination is $34^{\circ} \pm 5^{\circ}$ from face-on and the position angle of the major axis is $78^{\circ} \pm 10^{\circ}$ (east of north). For the outer ellipse the inclination is slightly higher with $47^{\circ} \pm 2^{\circ}$ while the position angle is almost unchanged with $82^{\circ} \pm 3^{\circ}$. The apparent discrepancy in the disk inclination for the two disk regions might be caused by a combination of a flared disk geometry (see Sects. 4.2 and 4.3) and the forward/backward scattering and polarization properties of the dust grains. A disk model, which is beyond the scope of the current paper, might help to shed light on this
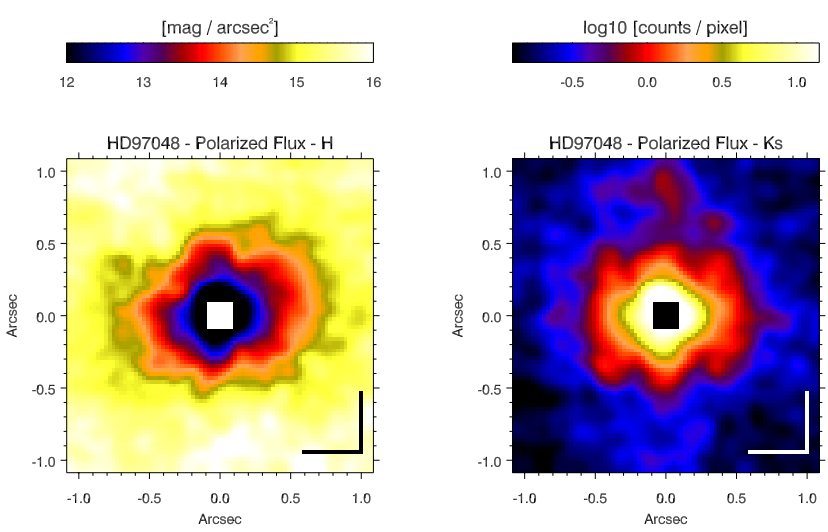

Fig. 2. Images showing the total polarized flux in the $H$ filter $(l e f t)$ and $K_{\mathrm{s}}$ filter $(r i g h t)$. The innermost saturated pixels were masked out and the images were convolved with a Gaussian kernel with a FWHM of 4 pixels (i.e., the FWHM of the PSF). While the $H$ image is flux-calibrated, showing the flux in units of surface brightness, the unit of the $K_{\mathrm{s}}$ image is counts per pixel. North is up, east to the left.

question. Because the $K_{\mathrm{s}}$-band image is not flux-calibrated, it is not possible to derive a disk inclination that is related to a certain apparent surface brightness level. However, a fit to disk regions that roughly correspond to the same spatial regions as the inner ellipse in the $H$-band yields an inclination and position angle that are identical to those derived for the $H$-band image within the error bars.

Knowing the inclination, one can determine the radial brightness profile of the disk along the major axis. Figure 3 shows the average radial brightness profile computed in a three-pixel wide box along both semi-major axes. For the orientation of the box we used the average position angle derived from the ellipse fitting exercise described above, i.e., $80^{\circ}$. The errors are the root-mean-square of the standard deviation within each box for a given separation and the flux variation observed along both semimajor axes. We then fitted a power-law of the form $S(r)=a r^{x}$ to these brightness profiles in both filters between $0.1^{\prime \prime}-1.0^{\prime \prime}$ (i.e., $\sim 16$ and $\sim 160 \mathrm{AU}$ projected separation). In the $H$ filter the power-law exponent is $-1.78 \pm 0.02$, for the $K_{\mathrm{s}}$ filter the exponent is $-2.34 \pm 0.04$. The fitting functions approximate the data reasonably well. Because the surface brightness decreases more steeply in the $K_{\mathrm{s}}$ band, the disk color $\left[H-K_{\mathrm{s}}\right]$ in polarized flux becomes bluer at larger separations, possibly indicating changes in the dust grain properties. Between $0.6^{\prime \prime}-0.8^{\prime \prime}$, however, the fits seem to slightly overestimate the measured profile in both filter. We will discuss this apparent "dip" in more detail in Sect. 4.1. 

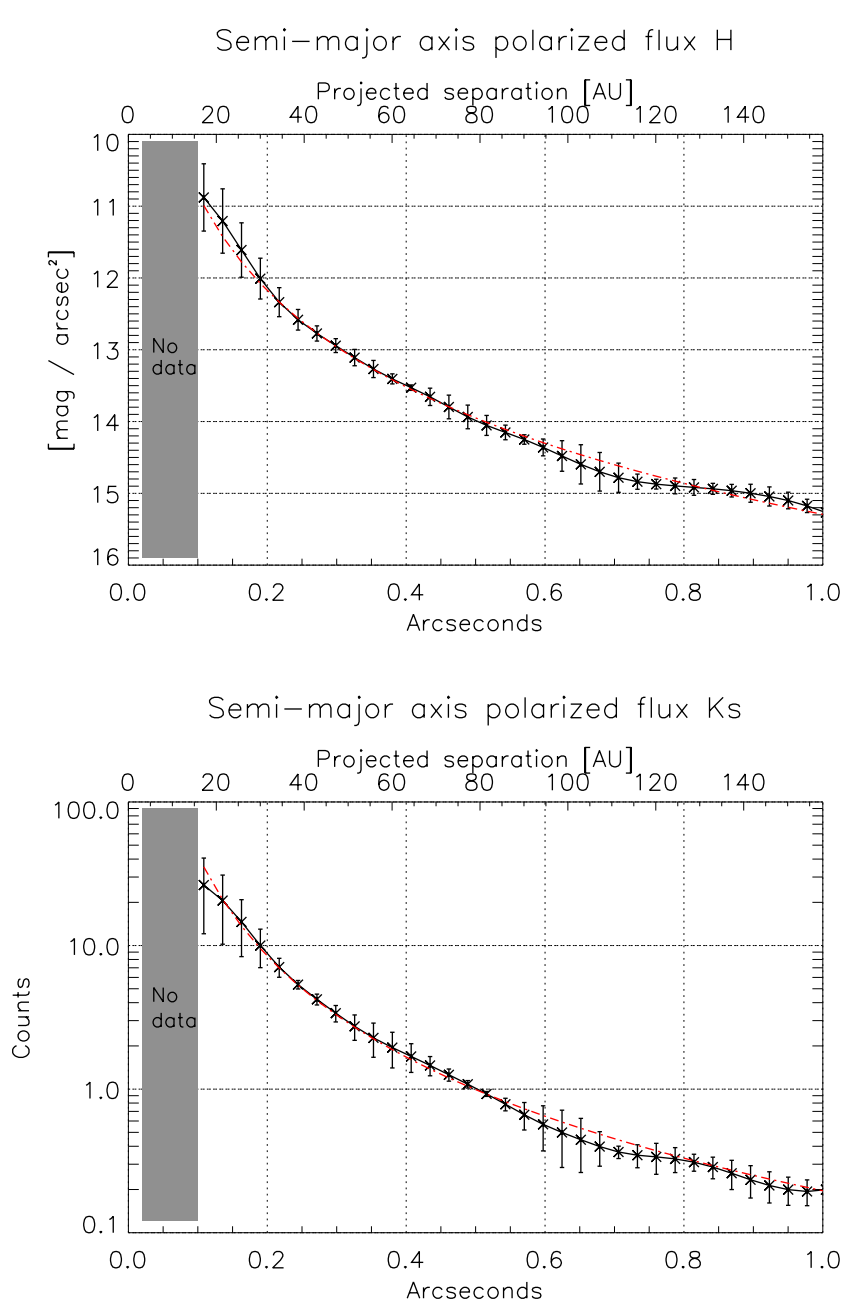

Fig. 3. Average surface brightness profiles along both semi-major axes of HD 97048 ( $H$ filter upper panel, $K_{\mathrm{s}}$ filter lower panel). The data are shown as black data points while the red, dashed lines are power-law fits to the data between $0.1^{\prime \prime}-1.0^{\prime \prime}$. The innermost saturated pixels were masked out, so there is not information available in the inner $0.1^{\prime \prime}$.

\section{Discussion}

\subsection{HD 97048 in polarized light}

The images presented here are the first resolved NIR scattered light images of the HD 97048 disk revealing the inner regions roughly between 16 and $160 \mathrm{AU}$ projected separation. It is interesting to note that the regions we observe here are on the same scale as the orbits of the three outer planets in the HR 8799 system ( 24-68 AU; Marois et al. 2008), in particular, because both objects are A-type stars.

As mentioned above, the surface brightness profiles of the HD 97048 disk contain some indications for a "dip" between $0.6^{\prime \prime}-0.8^{\prime \prime}$. In general, these dips in the observed radial brightness profiles could be caused by underlying physical gaps in the disk convolved to the spatial resolution of the observations. In our case, however, this apparent dip is more likely a result of small differences in the flux profiles along both semi-major axes. This also explains the larger error bars of the averaged radial profiles at these separations shown in Fig. 3.

\subsection{Comparison to HD 100546}

It is interesting to compare the results for HD 97048 presented here to those for HD 100546 presented in Quanz et al. (2011). Not only were both objects observed during the same observing run with the same instrumental setup, but both objects have a similar spectral type and both are members of the Herbig Be/Ae group I in the classification scheme introduced by Meeus et al. (2001). This means that the SED of both objects has a high MIR excess that is generally thought to arise from a flared disk geometry. In addition, both objects show strong PAH emission bands in the NIR and MIR (e.g., Van Kerckhoven et al. 2002; van Boekel et al. 2004, and references therein). However, there are also notably differences between the two objects. HD 100546 shows indications for a large gap in the disk between $\sim 4-15$ AU and it was speculated that a gas giant planet orbits in this gap (e.g. Bouwman et al. 2003; Acke \& van den Ancker 2006). Also, HD 97048 lacks a $10 \mu \mathrm{m}$ silicate emission feature in the MIR spectrum while HD 100546 has a very rich silicate band closely resembling that of comet Hale Bopp (Malfait et al. 1998; Bouwman et al. 2003; van Boekel et al. 2004). The lack of a silicate emission feature at $10 \mu \mathrm{m}$ on the one hand and the need for a strong opacity source that effectively absorbs in the UV/optical (making the disk flare) and re-emits strongly in the MIR continuum let van Boekel et al. (2004) to the conclusion that small carbonaceous grains are widely present in the HD 97048 disk.

Our data allow for the first time a direct comparison of the scattered light properties of the inner disk regions ( 20-140 AU) of HD 97048 and HD 100546. It shows that the radial brightness profile along the disk major axis is $\propto r^{-3}$ an thus steeper for HD 100546 compared to HD 97048 where we found roughly $\propto r^{-2}$. If the emission were optically thin and assuming everything else to be equal, this would indicate that the surface density of scattering dust particles is flatter (i.e., constant) on the surface of the HD 97048 disk and/or that dust composition is different. However, for an optically thick scattering surface the difference in the radial profiles can be explained with differences in the disk geometry, i.e., stronger disk flaring in HD 97048. And indeed, Benisty et al. (2010) was able to model the HD 100546 disk with a flaring index $\beta=0.5 \ldots 1.125$ between $13-350 \mathrm{AU}$, while Lagage et al. (2006) modeled the disk around HD 97048 with a flaring index of $\beta=1.26$ (see Sect. 4.3$)^{2}$.

A direct comparison between the $H$-band surface brightness of the two disks reveals that at $60 \mathrm{AU}$ both disks have roughly the same brightness with $13.5 \mathrm{mag} / \mathrm{arcsec}^{2}$. At smaller projected separations HD 100546 appears brighter, while at larger separations HD 97048 is brighter. Another way of comparing the surface brightness of the two disks is shown in Fig. 4, where we subtracted the observed $H$-band magnitude of the objects from the disk surface brightness in the $H$ band. The resulting brightness profiles are hence normalized with respect to the total $H$-band flux and provide some information about the general scattering efficiency of the dust. Interestingly, the plot shows that the normalized surface brightness of the the two disks is basically identical in the inner $20-40$ AU. At larger separations the disk of HD 97048 appears brighter because the surface brightness profile drops less rapidly (see above). For these analyses one has to keep in mind, though, that the absolute flux calibration for both sources suffers from uncertainties as described in Sect. 2.

\footnotetext{
2 The flaring geometry of a disk can be described by $H(r)=H_{0}\left(r / r_{0}\right)^{\beta}$ with $H(r)$ being the disk surface scale height as a function of the disk radius and $H_{0}$ and $r_{0}$ being the scale height and the disk radius at a reference point.
} 


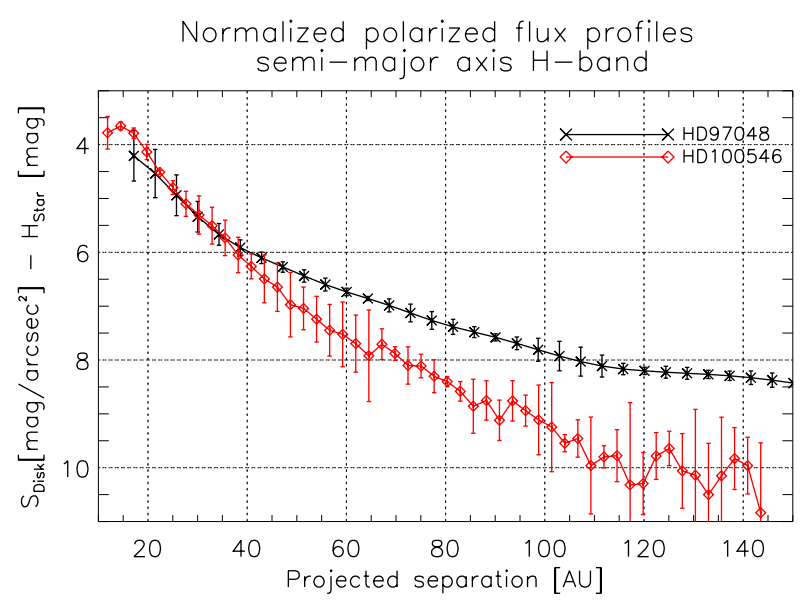

Fig. 4. Comparison of the normalized polarized flux profiles of HD 97048 (black crosses) and HD 100546 (red diamonds; Quanz et al. 2011) in the $H$ filter. For the normalization the observed $H$-band magnitude of the stars were subtracted from the surface brightness profiles of the disks. Both curves are the average of the observed surface brightness profiles along both semi-major axes.

Finally, we can compare the apparent color of the disk as a function of separation. While HD 100546 in general shows a red $\left[H-K_{\mathrm{s}}\right]$ color that becomes increasingly redder with increasing separation (Quanz et al. 2011), HD 97048 seems to become relatively bluer at larger separations (see Sect. 3). Concerning the size of the dust grains on the surface of the disk our data provide no immediate constraints for HD 97048.

\subsection{HD 97048 resolved in $P A H$, continuum, and gas emission}

Since this is the first time the inner disk regions of HD 97048 have been directly imaged in scattered light, we compared them with other observations probing various disk regions and components.

While earlier mid-infrared observations have shown that the emission of HD 97048 is extended on scales up to 5-10" and probably arises from an extended envelope surrounding the stardisk system (Prusti et al. 1994; Siebenmorgen et al. 2000), the first observational evidence that HD 97048 might be surrounded by a large, flared circumstellar disk was provided by MIR spectroscopy (van Boekel et al. 2004) and later confirmed by direct imaging in PAH filters resolving the disk at $8.6 \mu \mathrm{m}$ and $11.3 \mu \mathrm{m}$ (Lagage et al. 2006; Doucet et al. 2007). Lagage et al. (2006) was able to fit the observations with a flared circumstellar disk with an inclination of $\sim 43^{\circ}$ from face-on and flaring geometry described by $H_{0} \approx 51 \mathrm{AU}, r_{0}=135 \mathrm{AU}$ and $\beta=1.26$ (see footnote 1). While the outer disk radius was found to be at least $370 \mathrm{AU}$, no direct information was obtained for the inner disk regions $\left(\leq 0.5^{\prime \prime}\right.$ corresponding to $\left.\lesssim 80 \mathrm{AU}\right)$ in these MIR images. The disk inclination we found from our scattered light images roughly agrees with the value found by Lagage et al. (2006) but the position angle of the disk major axis is different. In Lagage et al. (2006) and Doucet et al. (2007) it seems as if the disk major axis is aligned with the north-south direction giving rise to the observed brightness asymmetry in east-west direction. As seen above, in our images the position angle is close to the eastwest direction. We note, however, that we are probing different spatial scales and that, overall, the brightness asymmetry in east-west direction is mostly confined to radial distances $>0.5^{\prime \prime}$ in the PAH filters and not to the MIR continuum (Doucet et al. 2007; van Boekel et al. 2004; Mariñas et al. 2011).

Habart et al. (2004) showed that emission features at 3.43 and $3.53 \mu \mathrm{m}$, which were attributed to hydrogenated diamonds (Van Kerckhoven et al. 2002; Habart et al. 2004; Guillois et al. 1999, and references therein), as well as the nearby continuum emission was slightly extended and most likely arising from the innermost $15 \mathrm{AU}$ of a circumstellar disk seen face-on.

Doering et al. (2007) used HST/ACS to image the circumstellar surrounding of HD 97048 in the $F 606 W$ (broad $V$ ) filter. They used the $1.8^{\prime \prime}$ occulting spot of HST/ACS and a reference star for PSF subtraction to reveal scattered light out to a radial distance of $\sim 4^{\prime \prime}$ in almost all directions. Since the inner $2^{\prime \prime}$ (in radius) were excluded from any analysis because of subtraction residuals, these data probe different scales than our observations. Doering et al. (2007) found an azimuthally averaged peak surface brightness of $19.6 \pm 0.2 \mathrm{mag} / \operatorname{arcsec}^{2}$ at $2^{\prime \prime}$ separation with a radial fall-off $\propto r^{-3.3 \pm 0.5}$. Because this fall-off is significantly steeper than the profiles we found in the inner $1^{\prime \prime}$, it seems likely that these data do not represent a simple continuation of our observations to larger radii but that actually different dust populations are probed and it remains unclear whether the HST/ACS images can be fully interpreted in the context of an inclined and flared disk or whether the surrounding envelope contributes to the observed properties.

Looking at the gaseous disk component, Acke \& van den Ancker (2006) found the [OI] emission line broad and double peaked most likely arising from disk regions between $0.8 \mathrm{AU}$ and $~ 50 \mathrm{AU}$. van der Plas et al. (2009) resolved several lines in the CO ro-vibrational emission band at $4.7 \mu \mathrm{m}$ consistent with being double-peaked and found that the emission extends from $11 \mathrm{AU}$ to at least $100 \mathrm{AU}$. The lack of emission closer to the star was taken as a possible sign of photo-dissociation of $\mathrm{CO}$ as [OI], and hence gas, seems to exist also in the inner 10 AU. Interestingly, Carmona et al. (2011) found that the $\mathrm{H}_{2} \quad 1-0 \mathrm{~S}(1)$ ro-vibrational line at $2.12 \mu \mathrm{m}$ was not only clearly detected but extended from $\sim 5-10$ AU out to $>200 \mathrm{AU}$. Photo-dissociation in the inner disk regions and heating by high-energy UV and X-ray photons was suggested as a possible origin for the observed emission. Note that also the $\mathrm{S}(1)$ pure rotational line of $\mathrm{H}_{2}$ at $17 \mu \mathrm{m}$ was detected but unresolved (Martin-Zaïdi et al. 2007, 2009).

This shows that the emitting regions of the gas emission lines are directly comparable to the regions probed by our data. In Fig. 5 we have sketched a cartoon of the HD 97048 disk showing what sort of spatially resolved information is available. Our NACO/PDI data nicely extend the existing resolved information from the PAH emission closer to the star and probe exactly those regions were several gas species have also been resolved spatially. This makes HD 97048 an ideal laboratory for sophisticated disk models studying both disk components - gas and dust - and their interplay with another and with the host star.

\section{Summary and conclusions}

For the first time the inner $\sim 0.1^{\prime \prime}-1.0^{\prime \prime}(\sim 16-160 \mathrm{AU})$ of the surface layer of the circumstellar disk around the Herbig Ae/Be star HD 97048 have been imaged in scattered light. At least one other early-type star is known to host three giant planets at separations comparable to the regions probed by our images (HR8799; Marois et al. 2008). Our data fill a gap in a large collection of existing observations of HD 97048, partly resolving the thermal dust emission as well as gas and PAH emission 


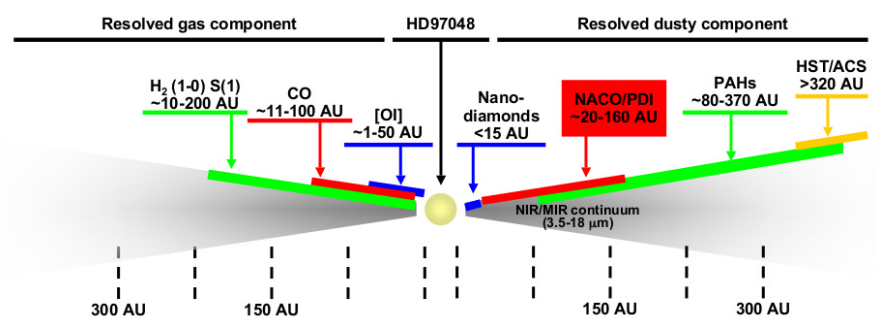

Fig. 5. Sketch summarizing the amount of spatially resolved data available for HD 97048. The gaseous disk component is shown on the lefthand side, while the dusty disk component is shown on the right-hand side. References for the different observations are given in the text. Our NACO/PDI data are indicated by the red box on the right-hand side.

lines on comparable scales. Fitting isophots to the flux-calibrated $H$-band images we found an apparent disk inclination of $34^{\circ} \pm 5^{\circ}$ and $47^{\circ} \pm 2^{\circ}$ for disk regions with a surface brightness between 13-14 mag/ $\operatorname{arcsec}^{2}$ and 14-15 mag/ $\operatorname{arcsec}^{2}$, respectively. For the brighter regions the position angle of the major axis is $78^{\circ} \pm 10^{\circ}$ (east of north), which remains almost unchanged going to the fainter regions $\left(82^{\circ} \pm 3^{\circ}\right)$. The surface brightness of the polarized flux drops from $\sim 11 \mathrm{mag} / \operatorname{arcsec}^{2}$ at $\sim 0.1^{\prime \prime}(\sim 16 \mathrm{AU})$ to $\sim 15.3 \mathrm{mag} / \operatorname{arcsec}^{2}$ at $\sim 1.0^{\prime \prime}(\sim 160 \mathrm{AU})$. Because the surface brightness decreases more rapidly in $K_{\mathrm{s}}$ compared to $H$, the disks becomes relatively bluer at larger separations, possibly indicating changing dust grain properties as a function of radius. The surface brightness profiles along the disk major axis can be fitted with power-laws falling off as $\propto r^{-1.78 \pm 0.02}$ in $H$ and $\propto r^{-2.34 \pm 0.04}$ in $K_{\mathrm{s}}$. In our images, we found no significant indications for additional disk structures, e.g., gaps.

The data presented here demonstrate that

1) polarimetric differential imaging (PDI) is a very powerful high-contrast technique to image dusty circumstellar disks at inner working angles where we know that planets can form. Other techniques such as classical PSF subtraction or coronagraphy are typically limited to larger separations because the inner disk regions (typically a few tens of AU) either suffer from subtraction residuals or are blocked out by the coronagraph.

2) Our detection of scattered light around HD 97048 makes this object a prime target for future follow-up observations with high-contrast instruments such as SPHERE (Beuzit et al. 2006) at the VLT or GPI (Macintosh et al. 2006) at Gemini. These new instruments with their high-performance AO systems will provide a much more stable PSF and are consequently better suited for high-contrast PDI observations than current instruments. Additionally, SPHERE has also a high-precision imaging polarimeter in the optical wavelength range (ZIMPOL; Roelfsema et al. 2010), complementing the high-contrast capabilities in the NIR. Potentially, SPHERE or GPI will not only confirm our detection but may provide new important insights into the dust grain properties, the disk structure, and the interplay between the spatially resolved gas and dust component in the inner regions of the HD 97048 circumstellar disk.

Acknowledgements. We are very grateful to the people who supported us during the observations, in particular Nancy Ageorges, Markus Hartung, and Nuria Huelamo. S.P.Q. thanks Vincent Geers for useful discussions about PAH emission and Hans Martin Schmid for providing feedback on an earlier version of this manuscript. We acknowledge support from the ESA/ESTEC Faculty Visiting Scientist Programme and thank the anonymous referee for useful comments.

\section{References}

Acke, B., \& van den Ancker, M. E. 2006, A\&A, 449, 267 Alibert, Y., Mordasini, C., \& Benz, W. 2011, A\&A, 526, A63 Apai, D., Pascucci, I., Brandner, W., et al. 2004, A\&A, 415, 671 Apai, D., Janson, M., Moro-Martín, A., et al. 2008, ApJ, 672, 1196 Benisty, M., Tatulli, E., Ménard, F., \& Swain, M. R. 2010, A\&A, 511, A75 Beuzit, J., Feldt, M., Dohlen, K., et al. 2006, The Messenger, 125, 29 Biller, B. A., Close, L. M., Masciadri, E., et al. 2007, ApJS, 173, 143 Borucki, W. J., Koch, D. G., Basri, G., et al. 2011, ApJ, 736, 19

Bouwman, J., de Koter, A., Dominik, C., \& Waters, L. B. F. M. 2003, A\&A, 401, 577

Carmona, A., van der Plas, G., van den Ancker, M. E., et al. 2011, A\&A, 533, A39

Chauvin, G., Lagrange, A., Bonavita, M., et al. 2010, A\&A, 509, A52

Cutri, R. M., Skrutskie, M. F., van Dyk, S., et al. 2003, 2MASS All Sky Catalog of point sources. (The IRSA 2MASS All-Sky Point Source Catalog, NASA/IPAC Infrared Science Archive, http://irsa.ipac.caltech. edu/applications/Gator/)

Doering, R. L., Meixner, M., Holfeltz, S. T., et al. 2007, AJ, 133, 2122

Doucet, C., Habart, E., Pantin, E., et al. 2007, A\&A, 470, 625

Guillois, O., Ledoux, G., \& Reynaud, C. 1999, ApJ, 521, L133

Habart, E., Testi, L., Natta, A., \& Carbillet, M. 2004, ApJ, 614, L129

Henning, T., Burkert, A., Launhardt, R., Leinert, C., \& Stecklum, B. 1998, A\&A, 336, 565

Janson, M., Bonavita, M., Klahr, H., et al. 2011, ApJ, 736, 89

Kasper, M., Apai, D., Janson, M., \& Brandner, W. 2007, A\&A, 472, 321

Kuhn, J. R., Potter, D., \& Parise, B. 2001, ApJ, 553, L189

Lafrenière, D., Doyon, R., Marois, C., et al. 2007, ApJ, 670, 1367

Lagage, P.-O., Doucet, C., Pantin, E., et al. 2006, Science, 314, 621

Lagrange, A., Gratadour, D., Chauvin, G., et al. 2009, A\&A, 493, L21

Lagrange, A.-M., Bonnefoy, M., Chauvin, G., et al. 2010, Science, 329, 57

Lenzen, R., Hartung, M., Brandner, W., et al. 2003, in SPIE Conf. Ser. 4841, ed. M. Iye, \& A. F. M. Moorwood, 944

Macintosh, B., Graham, J., Palmer, D., et al. 2006, in SPIE Conf. Ser., 6272

Malfait, K., Waelkens, C., Waters, L. B. F. M., et al. 1998, A\&A, 332, L25

Mariñas, N., Telesco, C. M., Fisher, R. S., \& Packham, C. 2011, ApJ, 737, 57

Marois, C., Macintosh, B., Barman, T., et al. 2008, Science, 322, 1348

Marois, C., Zuckerman, B., Konopacky, Q. M., Macintosh, B., \& Barman, T. 2010, Nature, 468, 1080

Martin-Zaïdi, C., Lagage, P.-O., Pantin, E., \& Habart, E. 2007, ApJ, 666, L117

Martin-Zaïdi, C., Habart, E., Augereau, J.-C., et al. 2009, ApJ, 695, 1302

Masciadri, E., Mundt, R., Henning, T., Alvarez, C., \& Barrado y Navascués, D. 2005, ApJ, 625, 1004

Mayor, M., Marmier, M., Lovis, C., et al. 2011, A\&A, submitted [arXiv: 1109.2497]

Meeus, G., Waters, L. B. F. M., Bouwman, J., et al. 2001, A\&A, 365, 476

Perrin, M. D., Graham, J. R., Kalas, P., et al. 2004, Science, 303, 1345

Potter, D. E. 2003, Ph.D. Thesis, University of Hawai'i

Prusti, T., Natta, A., \& Palla, F. 1994, A\&A, 292, 593

Quanz, S. P., Meyer, M. R., Kenworthy, M. A., et al. 2010, ApJ, 722, L49

Quanz, S. P., Schmid, H. M., Geissler, K., et al. 2011, ApJ, 738, 23

Roelfsema, R., Schmid, H. M., Pragt, J., et al. 2010, in SPIE Conf. Ser., 7735

Rousset, G., Lacombe, F., Puget, P., et al. 2003, in SPIE Conf. Ser. 4839, ed.

P. L. Wizinowich \& D. Bonaccini, 140

Siebenmorgen, R., Prusti, T., Natta, A., \& Müller, T. G. 2000, A\&A, 361, 258

van Boekel, R., Waters, L. B. F. M., Dominik, C., et al. 2004, A\&A, 418, 177

van den Ancker, M. E., The, P. S., Tjin A Djie, H. R. E., et al. 1997, A\&A, 324, L33

van den Ancker, M. E., de Winter, D., \& Tjin A Djie, H. R. E. 1998, A\&A, 330, 145

van der Plas, G., van den Ancker, M. E., Acke, B., et al. 2009, A\&A, 500, 1137 Van Kerckhoven, C., Tielens, A. G. G. M., \& Waelkens, C. 2002, A\&A, 384, 568

van Leeuwen, F. 2007, A\&A, 474, 653

Whittet, D. C. B., Kirrane, T. M., Kilkenny, D., et al. 1987, MNRAS, 224, 497

Whittet, D. C. B., Prusti, T., Franco, G. A. P., et al. 1997, A\&A, 327, 1194

Witzel, G., Eckart, A., Buchholz, R. M., et al. 2011, A\&A, 525, A130 